This is the final peer-reviewed accepted manuscript of:

D. Mazza, D. Tarchi and G. E. Corazza, "A Unified Urban Mobile Cloud Computing Offloading Mechanism for Smart Cities," in IEEE Communications Magazine, vol. 55, no. 3, pp. 30-37, March 2017.

The final published version is available online at DOI: 10.1109/MCOM.2017.1600247CM

Rights / License:

The terms and conditions for the reuse of this version of the manuscript are specified in the publishing policy. For all terms of use and more information see the publisher's website. 


\title{
A Unified Urban Mobile Cloud Computing Offloading Mechanism for Smart Cities
}

\author{
Daniela Mazza, Daniele Tarchi, Senior Member, IEEE, and \\ Giovanni E. Corazza, Senior Member, IEEE, \\ Department of Electrical, Electronic and Information Engineering \\ University of Bologna \\ 40136 Bologna, Italy
}

\begin{abstract}
The increasing urbanization level of the world population has driven the development of a Smart City geographic system, conceived as a fully connected wide area characterized by the presence of a multitude of smart devices, sensors and processing nodes aimed at distributing intelligence into the city. At the same time, the pervasiveness of wireless technologies has led to the presence of heterogeneous networks, operating simultaneously in the same city area. One of the main challenges in this context is to provide sustainable solutions able to jointly optimize the data transfer, exploiting heterogeneous networks, and the data processing, exploiting heterogeneous devices, for managing Smart City applications for the citizens community. In this paper, the Urban Mobile Cloud Computing (UMCC) framework is developed, introducing a mobile cloud computing model describing the flows of data and operations taking place in the Smart City. In particular, we focus on the proposal of a unified offloading mechanism where communication and computing resources are jointly managed allowing a load balancing among the different entities in the environment, delegating both communication and computation tasks in order to satisfy the Smart City application requirements. This allows to cope with the limited battery power and computation capacity of the Smart Mobile Devices (SMDs), and plays a key role in a smart environment where wireless communication is of utmost relevance, particularly in mobility and traffic control domains.
\end{abstract}

Index Terms

Smart City, Mobile Cloud Computing, HetNets, Offloading mechanisms, QoS management. 


\section{INTRODUCTION}

According to the World Urbanization Prospect ${ }^{1}$ published by the United Nations, more than half of the population is living nowadays in urban areas, and about $70 \%$ will be city persons by 2050. At the same time with urbanization, an extraordinary phenomenon concerning the Information and Communication Technology (ICT) is happening: according to the Visual Networking Index ${ }^{2}$, the number of connected devices in mobility has overtaken the number of people in the world, and by 2018 it will be over 10 billion, including Machine to Machine (M2M) modules in the Internet of Things. Mobile data traffic is expected to increase about 11 times in the next five years.

Urbanization and ICT expansions are finding a relevant convergence point in the Smart City concept, the icon of sustainable and livable city, projecting the ubiquitous and pervasive computing paradigms to urban spaces, focusing on developing city network infrastructures, optimizing traffic and transportation flows, lowering energy consumption and offering innovative services. It is through ICT that Smart Cities are truly turning smart [1], in particular exploiting smart mobile devices in a Mobile Cloud Computing (MCC) context [2]. However, the huge amount of data generated in a Smart City environment could be overwhelming, due to the rising and diversified Quality of Service (QoS) requirements of the city services in relation to the computation time and the energy consumed by the devices. In order to face the explosion of Big Data to be stored and elaborated in a Smart City, mobile devices need to be supported by cloud and fog computing structures [3], allowing an optimized load-sharing in the network for both data storage and processing features.

For this reason, a new urban framework, named Urban MCC (UMCC), is developed herein. While in [4]-[6] specific solutions were introduced and analyzed, here the full system view is provided with requirements and optimization framework. UMCC can be thought of as the technological nervous system, allowing the networks and information flows of the city to enjoy a better urban way of life. UMCC is composed by different network and computing elements, having heterogeneous requirements and capabilities. Within it, the offloading process emerges as the opportune method for balancing the workload in a twofold way: on one hand, networkoffloading [7] distributes data traffic among the different wireless access technologies within the

\footnotetext{
${ }^{1}$ http://esa.un.org/unpd/wup/

${ }^{2}$ http://www.cisco.com/c/en/us/solutions/service-provider/visual-networking-index-vni/index.html
} 
Heterogeneous Network (HetNet) environment. On the other hand, computation-offloading, or cyberforaging [8], delegates computing functions to the cloud. In this context, a novel unified offloading mechanism can be envisaged. By means of the UMCC framework, data can be stored and processed by resource-rich devices using a dynamic cell association for delegating workload, thus shortening execution time, extending battery life and exploiting the possibility to preserve data in the cloud. The proposed framework implements a unified offloading mechanism that allows to optimize the system, by offloading both communication and computing tasks in order to satisfy the Smart City application requirements.

The unified offloading operation, within the UMCC framework, can be driven by a purposely defined utility function where throughput, energy efficiency, latency and computing performance are taken into account. Several works have already analyzed the characteristics of MCC offloading. In Tab. I the strengths and weaknesses with respect to UMCC of some of the most important works are summarized.

[TABLE 1 about here.]

The rest of the paper is organized as follows. In the Section II, the main requirements of a Smart City environment are introduced, by focusing on some specific applications. In Section III, the proposed UMCC framework is introduced by focusing on the main constitutive entities, while in Section IV, the offloading mechanism taking advantage of the UMCC framework is discussed. Finally, in Section V, the conclusions are drawn.

\section{Requirements of SMART City APPLiCATIONS}

There are many taxonomies trying to define Smart City key areas, where social aims, care for environment, and economic issues are related and interconnected. The European Research Cluster on the Internet of Things (IERC) has identified in [9] a list of applications in different Internet of Things (IoT) domains, including the Smart City domain. Moreover, the Net!Works ETP has issued a white paper [10] aiming to identify the major topics of Smart Cities that will influence the ICT environment. Furthermore, a relevant document aiming to categorize and define the different applications has been released by the European Telecommunications Standards Institute (ETSI), where several application types have been specified focusing on their bandwidth requirements [11]. 
Taking into account all the relevant aforementioned essays, we selected some important Smart City applications in order to identify their requirements and, then, to leverage the UMCC, . Each application can be defined through the services provided to the citizens, concerning the requirements in terms of:

- Latency: the amount of time required by a certain application between the event happens and the event is acquired by the system;

- Energy Consumption: the energy consumed for executing a certain application locally or remotely;

- Throughput: the amount of bandwidth required by a specific application to be reliably executed in the Smart City environment;

- Computing: the amount of computing processes requested by a certain application;

- Exchanged data: the amount of input, output and code information to be transferred by means of the wireless network;

- Storage: the amount of storage space required for storing the sensed data and/or the processing application;

- Users: the number of users for achieving a reliable service.

The QoS of a certain application can be seen as a function, where each requirement plays a role less or more important depending on the application type. In the following, we list some of the most influential Smart City applications, by highlighting their technological requirements and characteristics, while, in Tab. II, the considered application types and the significance of their requirements are summarized.

[TABLE 2 about here.]

a) Mobility: All the components in an intelligent transportation system could be connected to improve transportation safety, relieve traffic congestion, reduce air pollution and enhance comfort of driving. The necessary throughput, the computational load and the amount of data to exchange are high, whereas we can think the storage as a secondary requirement, unless for security recording.

b) Healthcare: Intelligent and connected medical devices, monitoring physical activity and providing efficient therapy management by using patients' personal devices, could be connected to medical archives and provide information for medical diagnosis. In this case, there are 
relatively low requirements regarding energy consumption, throughput and number of users, whereas the requirements in terms of latency, computation, exchanged data and storage are high.

c) Disaster Recovery: In a disaster relief scenario people are facing with the destruction of the infrastructures and local citizens are asked to use their mobile phones to photograph the site. In this case there are relatively low requirements regarding throughput, whereas it is important to have a quick response and to save the energy of the devices.

d) Energy: Energy saving can take advantage from the cloud basically thanks to smart grid systems, aimed to transform the behavior of individuals and communities towards a more efficient and greener use of electric power.

e) Waste Management: Automatically generated schedules and optimized routes which take into account an extensive set of parameters could be planned not only looking at the current situation, but also considering the future outlook. We can expect non-restrictive requirements of latency and throughput, whereas resource-poor devices have to be taken into consideration. The requirements related to data to be exchanged, load of computation, storage and number of users are not critical.

f) Tourism: Augmented reality and social networks are the characteristics of applications that more take advantage from the cloud, that becomes also useful for mobile users sharing photos and video clips, tagging their friends in popular social networks. We can expect not-restrictive requirements of latency and throughput, whereas resource-poor devices have to be taken into consideration. There are a great amount of data to be exchanged, load of computation and storage and number of users are variable.

By comparing the above described applications, it is possible to highlight that a Smart City scenario is composed of several heterogeneous services with different requirements. However, it is possible to note that most of them require a high computational complexity and a very high amount of data to be exchanged in order to be executed. Moreover, it should be noticed, that in a Smart City scenario multiple services co-exist, increasing even more the system requirements. This is at the base of the proposed UMCC architecture that, taking benefit from a joint distributed computing and communication infrastructure, can be implemented through the use of heterogeneous cloud computing and wireless networks. 


\section{UMCC FRAMEWORK}

UMCC sprang from the MCC, that is gaining an increasing interest in the recent years, due to the possibility of exploiting both cloud computing and mobile devices for enabling a distributed cloud infrastructure [2]: on one hand, the cloud computing idea has been introduced as a mean for allowing a remote computation, storage and management of information, and, on the other hand, the mobility skill allows to gain by the most modern smart devices and broadband connections for creating a distributed and flexible virtual environment. At the same time, the recent advances in the wireless technologies are defining a novel pervasive scenario where several heterogeneous wireless networks interact among them, giving the users the ability to select the best radio access among those in a certain area. As a consequence, the development of UMCC is introduced, gaining from both computing and wireless communication technologies. In the following the three pillars at the basis of the proposed UMCC framework are discussed.

\section{A. Smart Mobile Devices (SMDs)}

By analyzing the technology systems underlying a smart city framework, mobile devices can be considered in a three-fold way:

- Sensors: They can acquire different types of data regarding the users and the environment, transmitting a large amount of information to the cloud in real time, by means of wireless communication systems.

- Nodes: They can form distributed mobile clouds where the neighboring mobile devices are merged for resource sharing, becoming integral part of the network.

- Outputs: They can make the citizens aware of results and able to decide consequently, or become actuators without need of human intervention.

To perform this triple role, mobile devices have to become part of an infrastructure that is constituted by different cloud topologies and, at the same time, have to exploit heterogeneous wireless link technologies, allowing to address the different requirements of a smart city scenario. This infrastructure starts from the concept of MCC, where the cloud works as a powerful complement to resource-constrained mobile devices.

\section{B. Cloud Topologies}

In relation to the previously described SMD's roles, we take into account various cloud topologies. This is a different categorization with respect to the classical as a Service taxonomy 
used for cloud computing, i.e., SaaS, PaaS and IaaS. It looks on the different interaction among the nodes that form the cloud, instead of the services provided by the cloud itself, so we can distinguish among centralized cloud, cloudlet, distributed mobile cloud and a combination of them, as shown in Fig. 1:

- Centralized Cloud: A centralized cloud provides the citizens to interact remotely, e.g., for accessing to open data delivered by the public administrations. It refers to the presence of a remote cloud computing infrastructure having a huge amount of storage space and computing power, virtually infinite, offering the major advantage of the elasticity of resource provisioning.

- Cloudlet: Cloudlets are fixed small cloud infrastructures installed between the mobile devices and the centralized cloud, limiting their exploitation to the users in a specific area. Their introduction allows to decrease the latency of the access to cloud services by reducing the transfer distance at the cost of using smaller and less powerful cloud devices.

- Distributed Mobile Cloud: A third configuration can address the issue of non persistent connectivity, whereas both the previous concepts must assume a durable state of connection. In a distributed mobile cloud the neighboring mobile devices are pooled together for resource sharing [12].

The proposed UMCC framework foresees the joint exploitation of the aforementioned topologies.

[Fig. 1 about here.]

\section{Heterogeneous Access Technologies}

One of the most actual trend in wireless networks is the presence of a heterogeneous access platform allowing to several types of devices with multiple network interfaces to select among them the most suitable. Such a forthcoming scenario, introducing a higher degree of pervasiveness, allows, especially in a Smart City scenario, to enable the access of a multitude of different devices, from the high-end broadband user devices to the narrowband M2M devices.

Such network deployment, comprised of a mix of low-power nodes underlying the conventional homogeneous macrocell network, by deploying additional small cells within the local-area range and bringing the network closer to users, can significantly boost the overall network capacity through a better spatial resource reuse. Inspired by the attractive features and potential advantages 
of HetNets, their development have gained much momentum in the wireless industry and research communities during the past few years towards the $5 \mathrm{G}$ concepts.

\section{Towards a unified offloading mechanism}

The UMCC approach foresees the definition of a scenario where smart city applications can exploit jointly the three cloud topologies, as shown in Fig. 2, by distributing and performing among the different parts composing the framework, and heterogeneous wireless network access technologies, deployed in the urban area. The application requested by a specific SMD, named as the Requesting Smart Mobile Device (RSMD), is partitioned and distributed among the different clouds using the available access networks or computed locally (Fig. 2).

[Fig. 2 about here.]

The main issue is that, for transferring data from the requesting mobile device to the selected cloud topology, a certain time is required. This mostly depends on some communication parameters of the selected access network, such as the end-to-end throughput, the amount of users, the QoS management of a certain transmission technology between the user device and each type of cloud processing unit. Moreover, the access networks themselves could be already used by SMDs belonging to the smart city scenario, as well as other devices using the wireless infrastructures. This involves the necessity of designing a proper offloading method that by modeling both computing and communication resources as a single unique resource allows to distribute the computing/communication load in a fair way among the different clouds and access networks.

Hence, when a RSMD needs to select the cloud infrastructures to be used for computing the smart city application, two main elements have to be taken into account:

- the processing and storage devices - smart mobiles, per se or together forming distributed mobile clouds, and cloud servers, constituting the cloudlets and the centralized cloud;

- the wireless transmission equipment, - different access networks entailing diverse transmission speeds in relation to their own channel capacity and to the number of linked devices.

In Fig. 2, the UMCC framework is sketched by representing the functional flows of the architecture. Whenever a smart city application has to be performed, the citizen within the UMCC can select among different MCC infrastructures, aiming to respect the requirements of 
the specific application depending on their features. The distribution depends on the application requirements, and the UMCC features.

Computation, storage, and transmission features: The features of the selected processing and storage devices, considered per se or in a group forming cloud/cloudlets, are:

- Processing Speed: the speed of a device or a group of devices for processing the applications;

- Storage Capacity: the amount of storage space provided by a device or a group of devices.

At the same time, the features of the transmission equipment to be taken into account are:

- Channel Capacity: The nominal bandwidth of a certain communication technology that can be accessed by a certain device;

- Priority/QoS management: The ability of a certain communication technology to manage different QoS and/or priority levels;

- Communication interfaces: The number of communication interfaces of each device, that impacts on the possibility of selecting among the available HetNets.

\section{UMCC OFFLOADING MODEL}

Let's focus on one RSMD running an application App, defined through the number of operation to be executed, $O$, the amount of data to be exchanged, $D$, and the amount of data to be stored, $S$. An application can be seen as a smart city service, that can be executed either locally or remotely by exploiting the cloud infrastructures. Furthermore, each application has many requirements regarding the QoS levels. Among others, the most important are:

- the maximum accepted latency $T_{A p p}$, intended as the interval between a task of the application is requested and its results are acquired,

- the minimum level of energy consumption $E_{A p p}$, that the RSMD necessarily uses for performing the application itself,

- the throughput $\eta_{A p p}$, intended as the minimum bandwidth that the application needs for being performed.

The first acting entity in the system is the RSMD, characterized by certain features that are involved in the offloading operation: the power to compute applications locally, $P_{l}$, the power used for transferring data towards clouds, $P_{t r}$, the power for idling during the computation in the cloud, $P_{i d}$, the computing speed to perform locally the computation, $f_{l}$, and its storage availability, $H_{l}$. Furthermore, also the time-varying position of the device plays an important role in the system interactions. 
The different types of clouds considered in the Smart City scenario are characterized by their computing speed to perform the computation, i.e., $f_{c c}$ for the centralized cloud and $f_{c l}$ for cloudlets. Additionally, while the storage availability of the centralized cloud can be considered infinite, therefore not constraining in the interaction, the storage availability $\mathrm{H}_{c l}$ of each cloudlet has to be taken in consideration.

The distributed cloud is a set of SMDs, each characterized by its specific features in the same way of the RSMD, even if the role played by the SMDs is not a request but a provision of service. Furthermore, we are considering the system from the point of view of the RSMD. Thus, the involved features are: connectivity, computation and storage for the data exchange, i.e. the computing speed $f_{M D}$, the storage availability $H_{M D}$, the position $\operatorname{pos}_{M D}(x, y)$, the throughput $\eta_{\mathrm{MD}}$, the number of devices that can be connected to each SMD $n_{\mathrm{MD}}$, and their coverage range $r_{\mathrm{MD}}$

While the connection to the cloudlets can be made only through the unique Access Point (AP) that can be considered built-in in each cloudlet, and the connection to the SMDs of the distributed cloud can be made directly, the nodes of the HetNet offer different alternatives to connect towards the centralized cloud. For each involved node it is possible to define the position of the node $\operatorname{pos}_{\text {Nod }}(x, y)$, the end-to-end throughput in bit per second between the user and the exploited node $\eta_{N o d}$, the number of devices available to connect $n_{N o d}$, and the range of availability of the node $r_{N o d}$.

Tab. III summarizes the entities and the characteristics above described. They are in a certain relationship due to some physical and logical bounds that are derived from the following considerations.

[TABLE 3 about here.]

In order to distribute the computing and communication loads among the different elements, the system has to evaluate which HetNet nodes, cloudlets, and SMDs are available. On one hand, there are $M$ available HetNet nodes Nod for the communication offloading towards the centralized cloud, and $N$ cloudlets $C_{c l}$ and $K$ SMDs, able to offer computation offloading capabilities to the RSMD. On the other hand the system has to distribute, by means of all these entities, different percentages $\alpha_{i}$ of operations $O, \beta_{i}$ of data $D$, and $\gamma_{i}$ of memory $S$, to all the available nodes, cloudlets and devices.

The requirements related to the applications, and the associated QoS, can be respected by 
optimizing the application partitioning and node/cloud association based on the features of the processing and storage devices and of the transmission devices introduced in Section III-D; this corresponds to design a unified offloading mechanism, that, by taking into account both computing and communication resources and their relationships, as listed in Tab. III, as a whole, can distribute the loads to the different devices of the environment.

In this context a utility function aiming to optimize the application dependent QoS can be introduced, acting as input for the offloading procedure by selecting the best cloud and communication infrastructures, as shown in Fig. 3. The model constraints are derived from the observation that the sum of the offloaded fractions must be equal to 1 , thus the optimization problem becomes:

$$
\begin{gathered}
\max _{\alpha_{X i}, \beta_{X i}}\left\{w_{E} f\left(E_{R S M D}\left(\alpha_{X i}, \beta_{X i}\right)\right)+w_{T} f\left(T_{R S M D}\left(\alpha_{X i}, \beta_{X i}\right)\right)+w_{\eta} f\left(\eta_{R S M D}\left(\alpha_{X i}, \beta_{X i}\right)\right)\right\} \\
\text { s.t. } \quad \alpha_{0}+\sum_{i=1}^{M} \alpha_{H N i}+\sum_{i=1}^{N} \alpha_{C L i}+\sum_{i=1}^{K} \alpha_{M D i}=1 \\
\sum_{i=1}^{M} \beta_{H N i}+\sum_{i=1}^{N} \beta_{C L i}+\sum_{i=1}^{K} \beta_{M D i}=1
\end{gathered}
$$

The above equation corresponds to maximize a utility function defined as a weighted sum of the functions related to the energy consumed, the time spent and the throughput achieved by the RSMD, with constraints the amount of operations and data to be shared among the different entities. By doing this the system performs a unified offloading mechanism by considering jointly the communication and computing resources. In particular, the overall throughput can be evaluated as the sum of the throughput values $\eta_{X i}$ achievable through each node of the scenario. The throughput $\eta_{X i}$ is related to the number of SMDs $n_{X i}$ connected to the $i^{t h}$ node and the channel capacity $B W_{X i}$ of the $i^{t h}$ node, and can be expressed by resorting to the Shannon Formula. With respect to the latency, it can be evaluated as the sum of the local computing, the data transfer time toward and from the cloud/cloudlets, and the idle time during the offloaded computation. With respect to the consumed energy, it can be derived from the latency, as the weighted sum of each latency components by the power consumed in each state.

In Fig. 3, the functional blocks of the UMCC offloading mechanism, based on a utility function optimization, are represented. On one hand, the smart city applications define specific requirements, while the cloud topologies in a certain scenario set their features. The utility function aims at selecting those cloud topologies and access networks that allow to respect the 
requirements by setting an optimized distribution of the application itself. The optimization of the partition and the node association will impact again on the UMCC features to be used by the other applications.

[Fig. 3 about here.]

The maximization of the introduced utility function could be a nontrivial optimization problem, depending on the considered number of applications and devices acting in the selected scenario. To this aim different methods to find an optimal, or sub-optimal solution, of the objective function can be employed.

a) A Greedy approach: If the offloading operation is advantageous with respect to the local computation, the cell association scheme allows to select the 'best' node from the list of those available; such list can be completed by each SMD that sort each possible access node based on a self calculated objective function [4]. If the offloading cost is lower than the cost for the local computation, the SMD will connect to the node which minimizes the cost function, otherwise it will compute locally the application.

b) A cluster based approach: The idea is to divide the urban area in subareas having range $r$; each SMD can share resources only with the other SMDs, cloudlets, and HetNet access points placed in the same subarea. This approach, even if sub-optimal, can simplify the problem by reducing the amount of concurrent devices that are involved in the offloading; in [5] a cluster based optimization model is proposed, where the cluster size plays a significant role for optimizing the problem while keeping low the complexity.

c) Biased Randomization: A different approach can be obtained by resorting to probabilistic algorithms based on biased-randomization techniques [6]. In this problem setting, the most promising node concerning the potential increase in system efficiency has to be selected. The biased-randomization techniques work by introducing a biased or oriented random effect on the possible solutions of a problem, allowing to choose the best solution from a set of possible alternatives that are close to the global optimal. In [6] a biased randomization algorithm is proposed, allowing to approach the optimal solution by gaining from a heuristic algorithm, hence keeping the complexity low while approaching to the optimal solution. In [6], it is also possible to note that such an approach is feasible from the implementation point of view allowing to have a quasi-optimal solution in a reduced amount of time. 


\section{Conclusions}

In this paper we developed the UMCC framework, a concept that supports the smart city vision for the optimization of the QoS of various types of smart city applications. By exploiting the heterogeneous types of applications and devices, typical of a Smart City environment, and from the heterogeneous computing and communication infrastructure that composes the technological nervous system of the Smart City, the proposed UMCC framework allows to optimize the system performance by respecting the application requirements by performing a suitable partial offloading mechanism. The performance shown in specific applications allows to be optimistic about the UMCC practical effectiveness.

\section{REFERENCES}

[1] M. Dohler, C. Ratti, J. Paraszczak, and G. Falconer, "Smart cities [guest editorial]," IEEE Commun. Mag., vol. 51, no. 6, pp. 70-71, Jun. 2013.

[2] H. T. Dinh, C. Lee, D. Niyato, and P. Wang, "A survey of mobile cloud computing: architecture, applications, and approaches," Wireless Communications and Mobile Computing, vol. 13, no. 18, pp. 1587-1611, Dec. 2013.

[3] F. Bonomi, R. Milito, P. Natarajan, and J. Zhu, "Fog computing: A platform for internet of things and analytics," in Big Data and Internet of Things: A Roadmap for Smart Environments, ser. Studies in Computational Intelligence, N. Bessis and C. Dobre, Eds. Springer International Publishing, 2014, vol. 546, pp. 169-186.

[4] D. Mazza, D. Tarchi, and G. E. Corazza, "A user-satisfaction based offloading technique for smart city applications," in Proc. of IEEE Globecom 2014, Austin, TX, USA, Dec 2014.

[5] D. Mazza, D. Tarchi, and G. E. Corazza, "A cluster based computation offloading technique for mobile cloud computing in smart cities," in Proc. of IEEE ICC 2016, Kuala Lumpur, Malaysia, May 2016.

[6] D. Mazza, A. Pagès-Bernaus, D. Tarchi, A. A. Juan, and G. E. Corazza, "Supporting mobile cloud computing in smart cities via randomized algorithms," IEEE Systems Journal, 2016, accepted for publication.

[7] A. Aijaz, H. Aghvami, and M. Amani, "A survey on mobile data offloading: technical and business perspectives," IEEE Wireless Commun., vol. 20, no. 2, pp. 104-112, Apr. 2013.

[8] X. Ma, Y. Zhao, L. Zhang, H. Wang, and L. Peng, "When mobile terminals meet the cloud: computation offloading as the bridge," IEEE Netw., vol. 27, no. 5, pp. 28-33, Sep./Oct. 2013.

[9] O. Vermesan and P. Friess, Internet of Things - From Research and Innovation to Market Deployment. Aalborg, DK: River Publishers, 2014.

[10] L. M. Correia and K. Wunstel, "Smart cities application and requirements," White Paper, Net!Works European Technology Platform, May 2011.

[11] Electromagnetic compatibility and Radio spectrum Matters (ERM); System Reference document (SRdoc): Spectrum Requirements for Short Range Device, Metropolitan Mesh Machine Networks (M3N) and Smart Metering (SM) applications, ETSI Std. TR 103 055, 2011.

[12] D. Huang, T. Xing, and H. Wu, "Mobile cloud computing service models: a user-centric approach," IEEE Network, vol. 27, no. 5, pp. 6-11, Sep. 2013. 
[13] Y. Xu and A. Helal, "Scalable cloud-sensor architecture for the internet of things," IEEE Internet of Things Journal, vol. 3, no. 3, pp. 285-298, Jun. 2016.

[14] F. H. Bijarbooneh, W. Du, E. C. H. Ngai, X. Fu, and J. Liu, "Cloud-assisted data fusion and sensor selection for internet of things," IEEE Internet of Things Journal, vol. 3, no. 3, pp. 257-268, Jun. 2016.

[15] M. Jo, T. Maksymyuk, B. Strykhalyuk, and C. H. Cho, "Device-to-device-based heterogeneous radio access network architecture for mobile cloud computing,” IEEE Wireless Communications, vol. 22, no. 3, pp. 50-58, Jun. 2015.

Daniela Mazza received the masters degrees in electronic engineering and communication science and the $\mathrm{Ph} . \mathrm{D}$. degree in electronics, telecommunications, and information technologies engineering from the University of Bologna, Bologna, Italy. She is an expert on system optimization in public administration organizational contexts at Emilia Romagna Local Government, Italy. Her research interests include the services to the citizens, including solution for distributed multimedia system and services management in a context of smart city. She possesses more than 15 years of experience in the local government organizations and has worked as a Project Manager for technological packaging industries since 1991.

Daniele Tarchi (S'99, M'05, SM'12) received the M.Sc. degree in telecommunications engineering and the Ph.D. degree in informatics and telecommunications engineering from the University of Florence, Florence, Italy, in 2000 and 2004 , respectively. He is currently an Assistant professor at the University of Bologna, Bologna, Italy. His research interests include the telecommunication area, with particular interests to resource allocation and link adaptation algorithms in wireless and satellite networks. He has been involved in several national projects as well as European projects and has been active in several industry funded projects. Dr. Tarchi is an Editorial Board Member for the IEE Communications and for the IEEE Transactions on Vehicular Technology, and has been an Editorial Board Member for the Wiley Wireless Communication and Mobile Computing, Hindawi Journal of Engineering, and the Scientific World Journal and has served as an Associate Editor for the IEEE Transactions on Wireless Communications. He was Symposium Chair at IEEE Wireless Communications and Networking Conference 2011 and at IEEE Globecom 2014, and Workshop Co-Chair at the IEEE International Conference on Communications 2015. 
Giovanni Emanuele Corazza (M'92, SM'07) is currently a Full Professor with the Alma Mater Studiorum, University of Bologna, Bologna, Italy, a Member of the Alma Mater Board of Directors, the Founder of the Marconi Institute for Creativity (2011), a Member of the Marconi Society Board of Directors, a Member of the Board of the 5G Infrastructure Association, the Vice-Chairman of the NetWorld2020 European Technology Platform, and the founder of the Mavigex S.r.l. spin-off company. He was Head of the Department of Electronics, Computer Science and Systems (DEIS) in 20092012, the Chairman of the School for Telecommunications in 20002003, the Chairman of the Advanced Satellite Mobile Systems Task Force (ASMS TF), the Founder and Chairman of the Integral Satcom Initiative, a European technology platform devoted to satellite communications. He has authored or co-authored more than 260 papers. His research interests include wireless and satellite communications, mobile radio channel characterization, Internet of Things, navigation and positioning, estimation and synchronization, spread spectrum and multicarrier transmission, and scientific creative thinking. Prof. Corazza served as an Editor for Communication Theory and Spread Spectrum for the IEEE Transactions on Communications in 19972012. He was the recipient of the Marconi International Fellowship Young Scientist Award in 1995, the IEEE 2009 Satellite Communications Distinguished Service Award, the 2013 Newcom\# Best Paper Award, the 2002 IEEE Vehicular Technology Society Best System Paper Award, the Best Paper Award of the IEEE International Symposium on Spread Spectrum Techniques and Application (ISSSTA) 1998, at the IEEE International Conference on Telecommunication 2001, and at the 2nd International Symposium on. Wireless Communication Systems 2005. He was the General Chairman of the IEEE ISSSTA 2008, ASMS 20042012 Conferences, and MIC Conference 2013. 


\section{LIST OF FIGURES}

1 Cloud topologies in the UMCC framework: centralized cloud, cloudlet and distributed mobile cloud. . . . . . . . . . . . . . . . . . . 16

2 The process of distributing and performing the application among different parts of the UMCC . . . . . . . . . . . . . . . . . . . . . . . . 17

3 The utility function acts for distributing and performing the application in different parts of the Urban MCC . . . . . . . . . . . . . . . . . . . 18 


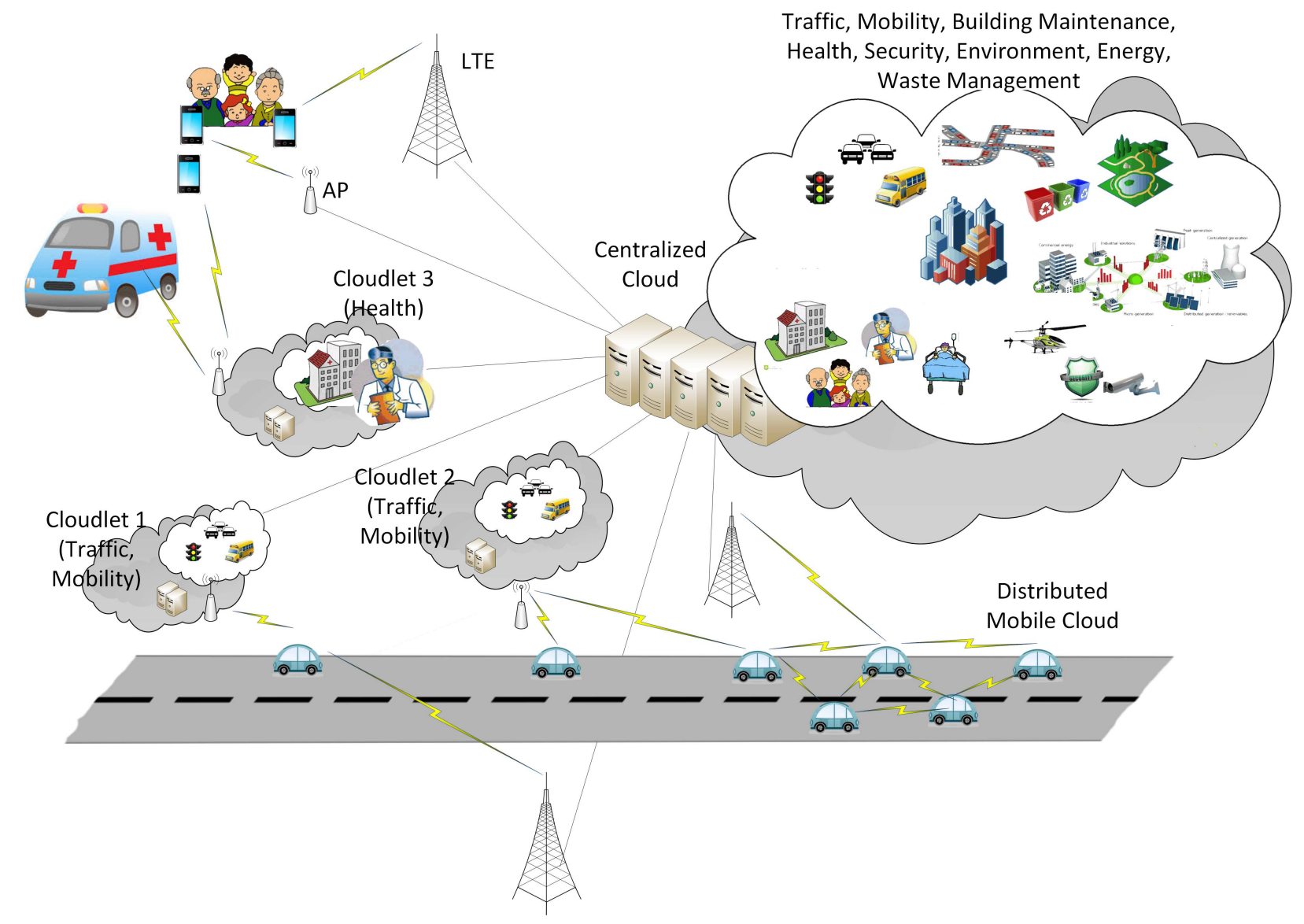

Fig. 1. Cloud topologies in the UMCC framework: centralized cloud, cloudlet and distributed mobile cloud. 


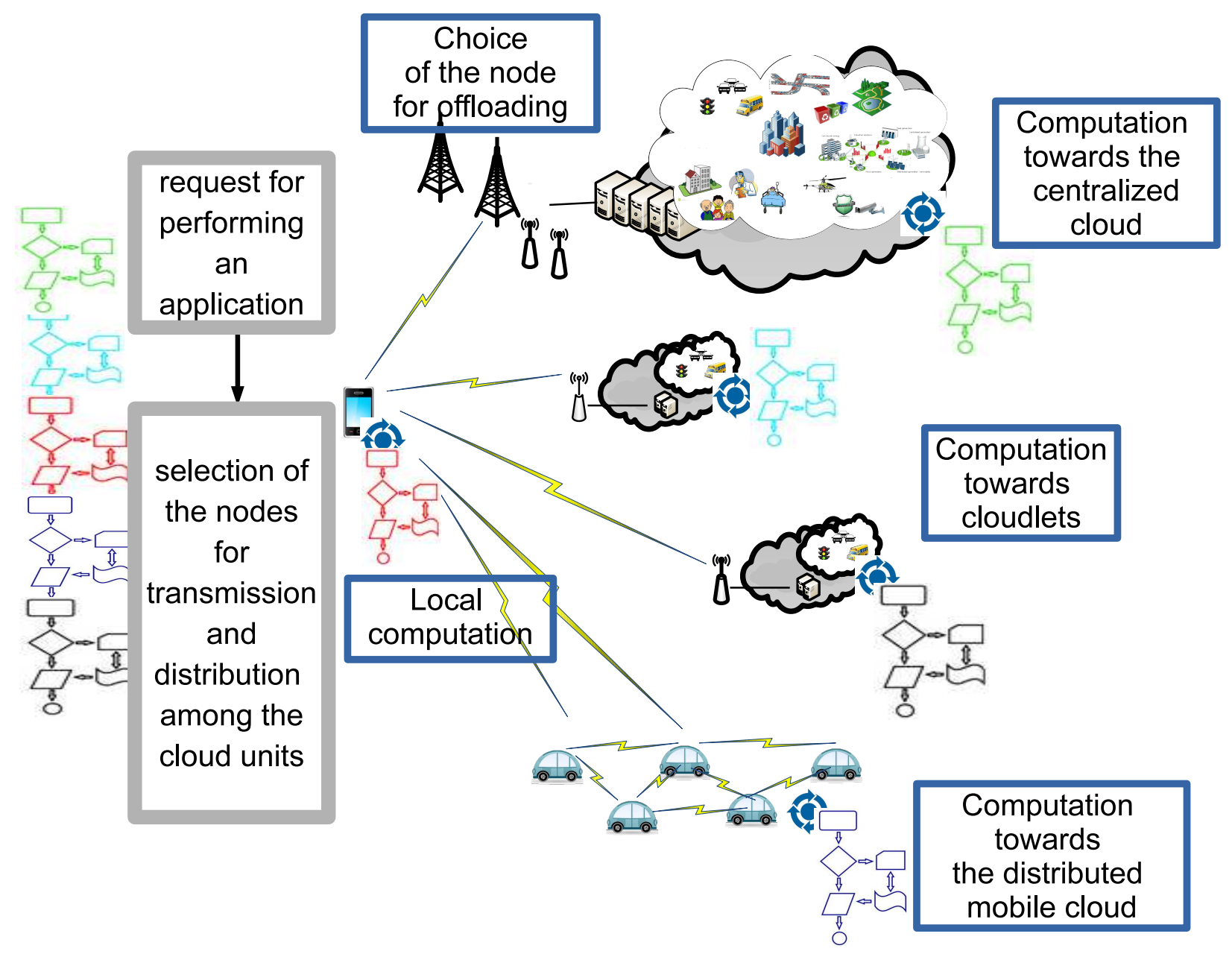

Fig. 2. The process of distributing and performing the application among different parts of the UMCC. 


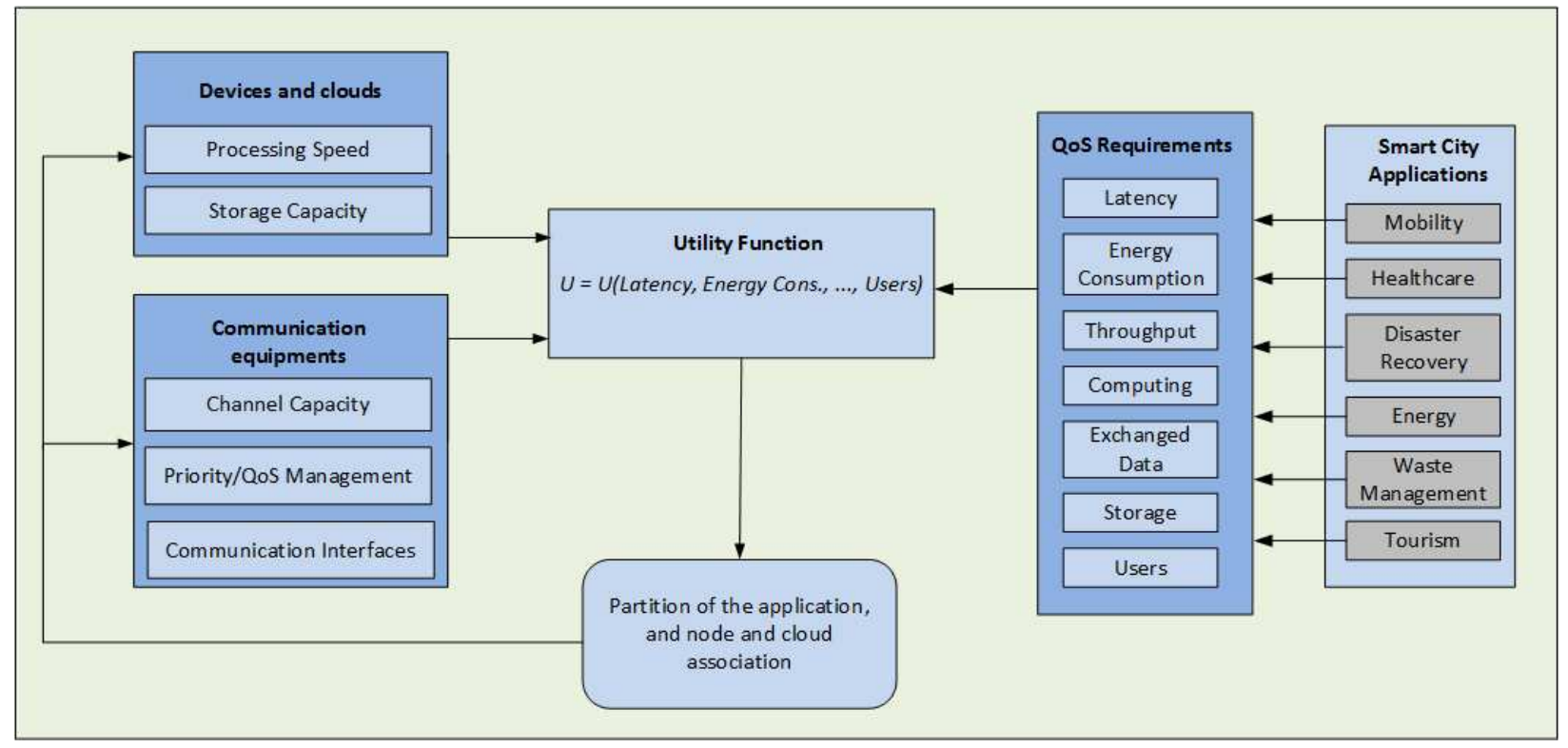

Fig. 3. The utility function acts for distributing and performing the application in different parts of the Urban MCC. 


\section{LIST OF TABLES}

I State of the Art summary . . . . . . . . . . . . . . . . . . . . 20

II Summary of Smart City applications and Requirements . . . . . . . . . . . 21

III Summary of entities and relations in the UMCC - Involved features and requirements 22 
TABLE I

STATE OF THE ART SUMMARY

\begin{tabular}{|c|c|c|c|}
\hline Reference & Objective & Strengths & Weaknesses w.r.t UMCC \\
\hline [13] & Cloud-Edge-Beneath (CEB) architecture & $\begin{array}{l}\text { Scalable ecosystem useful for } \\
\text { Smart City's massive scale of } \\
\text { devices }\end{array}$ & $\begin{array}{l}\text { No utility function definition, } \\
\text { by focusing mainly on archi- } \\
\text { tectural aspects }\end{array}$ \\
\hline$[14]$ & Cloud Assisted Data Fusion & $\begin{array}{l}\text { Efficient selection of nodes } \\
\text { with respect to link quality }\end{array}$ & $\begin{array}{l}\text { Mainly focused on data- } \\
\text { collection, while distributed } \\
\text { computing is not considered }\end{array}$ \\
\hline$[15]$ & Device To Device Based architecture & $\begin{array}{l}\text { Adds D2D communication to } \\
\text { cloud, with increased traffic } \\
\text { capacity }\end{array}$ & $\begin{array}{l}\text { No utility function definition, } \\
\text { by focusing mainly on the in- } \\
\text { creased global traffic, and not } \\
\text { on distributed computation }\end{array}$ \\
\hline$[12]$ & Mobile as a Representer (MaaR) & $\begin{array}{l}\text { User-centric characterization } \\
\text { using proactive behaviour }\end{array}$ & $\begin{array}{l}\text { No mathematical analysis, by } \\
\text { giving mainly an holistic per- } \\
\text { spective }\end{array}$ \\
\hline
\end{tabular}


TABLE II

SUMMARY OF SMART CITY APPLICATIONS AND REQUIREMENTS

\begin{tabular}{c||c|c|c|c|c|c|c|}
\multicolumn{1}{c||}{} & \multicolumn{7}{c|}{ Requirements } \\
\hline \multicolumn{1}{c||}{ Application } & latency & energy & throughput & computing & exchanged data & storage & users \\
\hline Mobility & restrictive & variable & restrictive & high & high & variable & high \\
Healthcare & restrictive & non-restrictive & non-restrictive & high & high & high & low \\
Disaster Recovery & restrictive & restrictive & non-restrictive & high & high & high & variable \\
Energy & non-restrictive & non-restrictive & non-restrictive & high & high & high & high \\
Waste Management & non-restrictive & restrictive & non-restrictive & low & low & low low \\
Tourism & non-restrictive & restrictive & non-restrictive & high & high & high variable \\
\hline
\end{tabular}


TABLE III

SUMMARY OF ENTITIES AND RELATIONS IN THE UMCC - INVOLVED FEATURES AND REQUIREMENTS

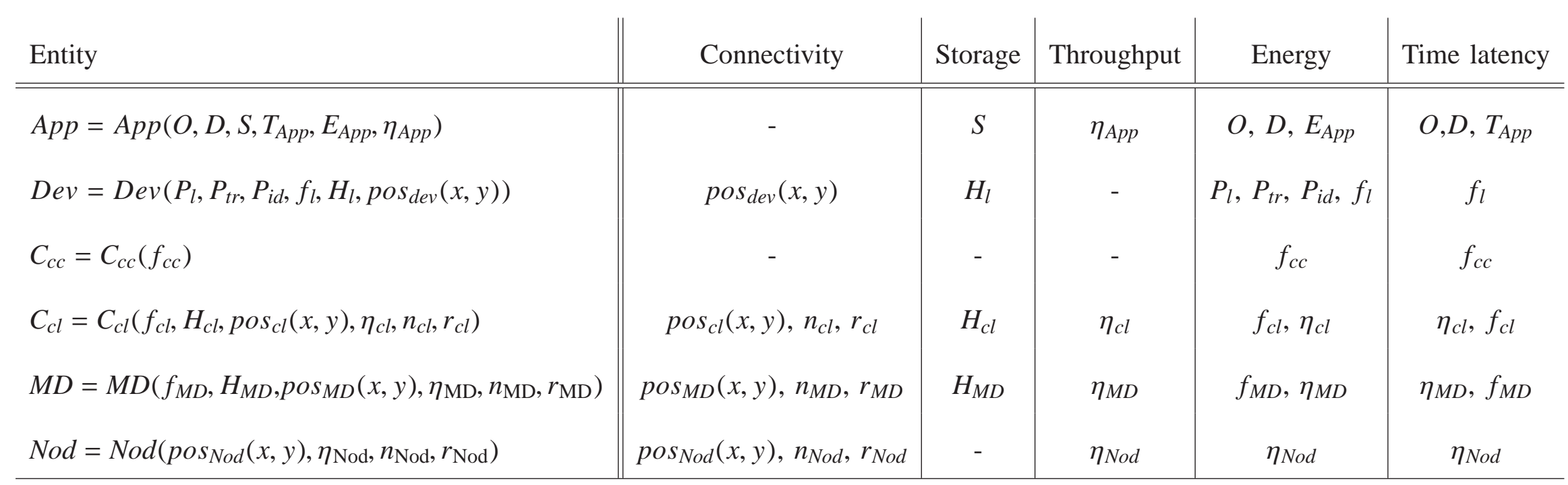

